\title{
NUEVOS REGISTROS FÓSILES DE VERTEBRADOS TERRESTRES PARA LAS LLANADAS, SANCTI SPÍRITUS, CUBA
}

\author{
Ernesto Aranda ${ }^{1}$, Joao G. Martínez López ${ }^{1}$, Osvaldo Jiménez ${ }^{2}$, Carlos Alemán Luna ${ }^{3}$ \\ y Lázaro W. Viñola López ${ }^{4}$ \\ 1. Museo Nacional de Historia Natural de Cuba. earanda@mnhnc.inf.cu; jgml@mnhnc.inf.cu. \\ 2. Gabinete de Arqueología de la Oficina del Historiador de la Ciudad de La Habana \\ osvaldojimenez@patrimonio.ohc.cu. \\ 3. Departamento de Estudios de Paleontología y Arqueología, Centro de Servicios Ambientales, \\ Sancti Spíritus, Cuba. \\ 4. Departamento de Ciencias de la Tierra, Universidad de Montana, Bozeman, \\ MT, 59717, EUA.1wv194@gmail.com
}

\section{RESUMEN}

El depósito paleontológico Las Llanadas es un manantial con sedimento rico en materia orgánica. Estudios del sitio a principios del siglo XX, dieron a conocer materiales fósiles de perezosos, cocodrilos, tortugas terrestres, y jutías. Dos especies de perezosos, Acratocnus antillensis y Neocnus gliriformis, fueron nuevas para la ciencia. En el presente trabajo se dan a conocer nuevos registros fósiles de anuros, un reptil y aves para el depósito: Peltophryne empusus, Osteopilus septentrionalis, Cyclura nubila, Tyto alba, Buteogallus borrasi y Boromys ofella. Huesos recientes de Lithobates catesbeianus y Capromys pilorides se encontraron entre los materiales, lo que indica que el depósito se mantiene activo en la recepción de especímenes. De un total de 2030 piezas solo 3\% pudo ser identificado taxonómicamente, debido al alto nivel de fragmentación.

Palabras clave: fósiles, vertebrados, cuaternario, Cuba.

\section{NEW FOSSIL RECORDS OF TERRESTRIAL VERTEBRATES FOR LAS LLANADAS, SANCTI SPÍRITUS, CUBA}

\begin{abstract}
The paleontological deposit Las Llanadas is a spring rich in organic matter. At the beginning of the 20th century, studies of the site reports fossil materials of sloths, crocodiles, tortoises, and hutias. Two species of sloths, Acratocnus antillensis and Neocnus gliriformis, were new to science. In the present work, we present new fossil records of Peltophryne empusus, Osteopilus septentrionalis (anurans), Cyclura nubila (reptile), Tyto alba, Buteogallus borrasi (birds), and Boromys ofella (mammal). Recent bones of Lithobates catesbeianus and Capromys pilorides were found among the fossils. Of 2030 pieces, only $3 \%$ could be identified taxonomically, due to the high level of fragmentation.
\end{abstract}

Keywords: fossil, vertebrates, Quaternary, Cuba.

\section{INTRODUCCIÓN}

Las Llanadas es uno de los depósitos paleontológicos cubanos más importantes del cuaternario. A comienzos del siglo XX los naturalistas Carlos de la Torre y Barnum Brown realizaron excavaciones y colectas de vertebrados fósiles en este sitio (Torre, 1910; Brown, 1913). 
Ellos encontraron restos de reptiles como la tortuga terrestre Chelonoidis [Testudo] cubensis y el cocodrilo cubano Crocodylus rhombifer [C. pristinus]; pero sin dudas la mayor importancia se debe a que Las Llanadas es la localidad tipo de las especies de perezosos Acratocnus antillensis y Neocnus gliriformis (Matthew, 1931).

Además de las especies mencionadas, en una visita a la colección herpetológica de la División de Paleontología del Museo Americano de Historia Natural, uno de los autores reconoció vértebras de la boa cubana Chilabothrus angulifer, así como un diente y una vértebra de la iguana Cyclura nubila, materiales de las excavaciones de Torre y Brown depositados como no identificados.

Este depósito paleontológico es un sitio abierto, dentro de una fisura de disolución cársica, localizada en la Sierra de Meneses y Cueto (Lomas de la Canoa), Norte de la Sierra de Jatibonico. Tiene dos áreas principales: una fisura cársica abierta, con dos paredes verticales (7 metros de altura), separadas por un pasillo estrecho (área 1); y un canal techado con una pequeña entrada (área 2). Ambas áreas se formaron por procesos de disolución del agua superficial y subterránea (Martínez-López, 2013).

En expedición realizada en abril del 2013, se extrajeron nuevos materiales paleontológicos del área 1, entre ellos se reconocieron todos los táxones registrados anteriormente y otros que constituyen nuevos registros. Las identificaciones se basaron en comparaciones anatómicas cualitativas con las colecciones del Museo Nacional de Historia Natural de Cuba (MNHNC), del Instituto de Ecología y Sistemática (IES), y la colección particular de Lázaro William Viñola (CLV). Todos los materiales están guardados en el MNHNC.

Cantidad, conservación, e identificación. Alrededor de 2030 piezas fueron encontradas, la mayoría de ellas fragmentadas $(97.7 \%)$ en pequeños pedazos con bajo o ningún valor taxonómico. Falanges, vértebras, astrágalos y calcáneos forman la mayor parte de las piezas conservadas, huesos con alta durabilidad tafonómica (Fernández-López, 2000). Todos los componentes esqueléticos aparecieron desarticulados y dispersos, lo que corresponde con la clase tafonómica III para el patrón de transporte y deposición descrita por Holz y Barberena (1994).

Todos los grupos de vertebrados, excepto peces, están presentes en la muestra. Los restos de algunos táxones tienen baja frecuencia de aparición, como es el caso del murciélago, de anuros, y aves rapaces; mientras que otros como los de cocodrilos, tortuga, jutías y perezosos son abundantes (Tabla I). Entre los perezosos están presentes las cuatro especies descritas para el Cuaternario cubano: Megalocnus rodens, Parocnus browni, Acratocnus antillensis, y Neocnus gliriformis.

El depósito contiene materiales en diferentes estados de fosilización, quizás por el tiempo de estancia o por las condiciones del medio. Incluso aparecen materiales recientes, como es el caso de un ilion de Lithobathes catesbeianus (una especie introducida), trozos de madera y conchas decoloridas de moluscos terrestres. Estos hallazgos indican que el depósito se mantiene activo en la asimilación, almacenamiento, y procesamiento de restos. 


\section{CLASIFICACIÓN \\ Clase Amphibia Linnaeus, 1758 \\ Orden Anura Fischer von Waldheim, 1813 \\ Familia Bufonidae Gray, 1825}

Peltophryne cf. empusus Cope, 1862 (fig.1A)

Fósil: un maxilar izquierdo.

Estado: viviente.

Familia Hylidae Rafinesque, 1815

Osteopilus septentrionalis Duméril y Bibron, 1841 (fig.1B)

Fósil: una mitad proximal de fémur, y un frontoparietal fragmentado.

Estado: viviente.

Clase Reptilia Laurenti, 1768

Orden Squamata Opel, 1811

Familia Iguanidae Gauther, 1948

Cyclura sp. Harlan, 1825 (fig.1C)

Fósil: un frontal y una vértebra.

Estado: viviente.

Familia Boidae Gray, 1825

Chilabothrus sp. Dumeril y Bibron, 1844 (fig.1D)

Reciente: dos vértebras.

Fósil: seis vértebras.

Estado: viviente.

Familia Dipsadidae Bonaparte, 1838

Cubophis sp. Hedges y Vidal, 2009 (fig.1F)

Reciente: una vértebra.

Estado: viviente.

Familia Tropidophiidae Brongersma, 1951

Tropidophis sp. Bibron, 1843 (fig.1G)

Fósil: dos vértebras.

Estado: viviente. 
Clase Aves Linnaeus, 1758

Orden Strigiformes Wagler, 1830

Familia Tytonidae Ridgway, 1914

Tyto alba Scopoli, 1769 (fig.1H)

Fósil: un fragmento proximal de tarsometatarso.

Estado: viviente.

Orden Falconiformes Sharpe, 1874

Familia Accipitridae Vieillot, 1816

Buteogallus sp. Arredondo, 1970 (fig.1E)

Fósil: dos falanges.

Estado: extinto.

Ave rapaz grande indeterminada

Fósil: una ulna fragmentada.

Estado: extinto.

Clase Mammalia Linnaeus, 1758

Orden Chiroptera Blumbenbach, 1779

Fósil: una ulna fragmentada.

Orden Rodentia Bowdich, 1821

Familia Echimyidae Gray, 1825

Boromys offella Miller, 1916 (fig.1I)

Fósil: una hemimandíbula izquierda.

Estado: extinto.

Otros restos. Alrededor de 20 coprolitos se encontraron junto a los restos de huesos, por su forma aún no se determina si fueron generados por cocodrilos o por perezosos gigantes. Dada su importancia en el estudio de la ecología de las especies, se están llevando a cabo análisis químicos para determinar su composición. 

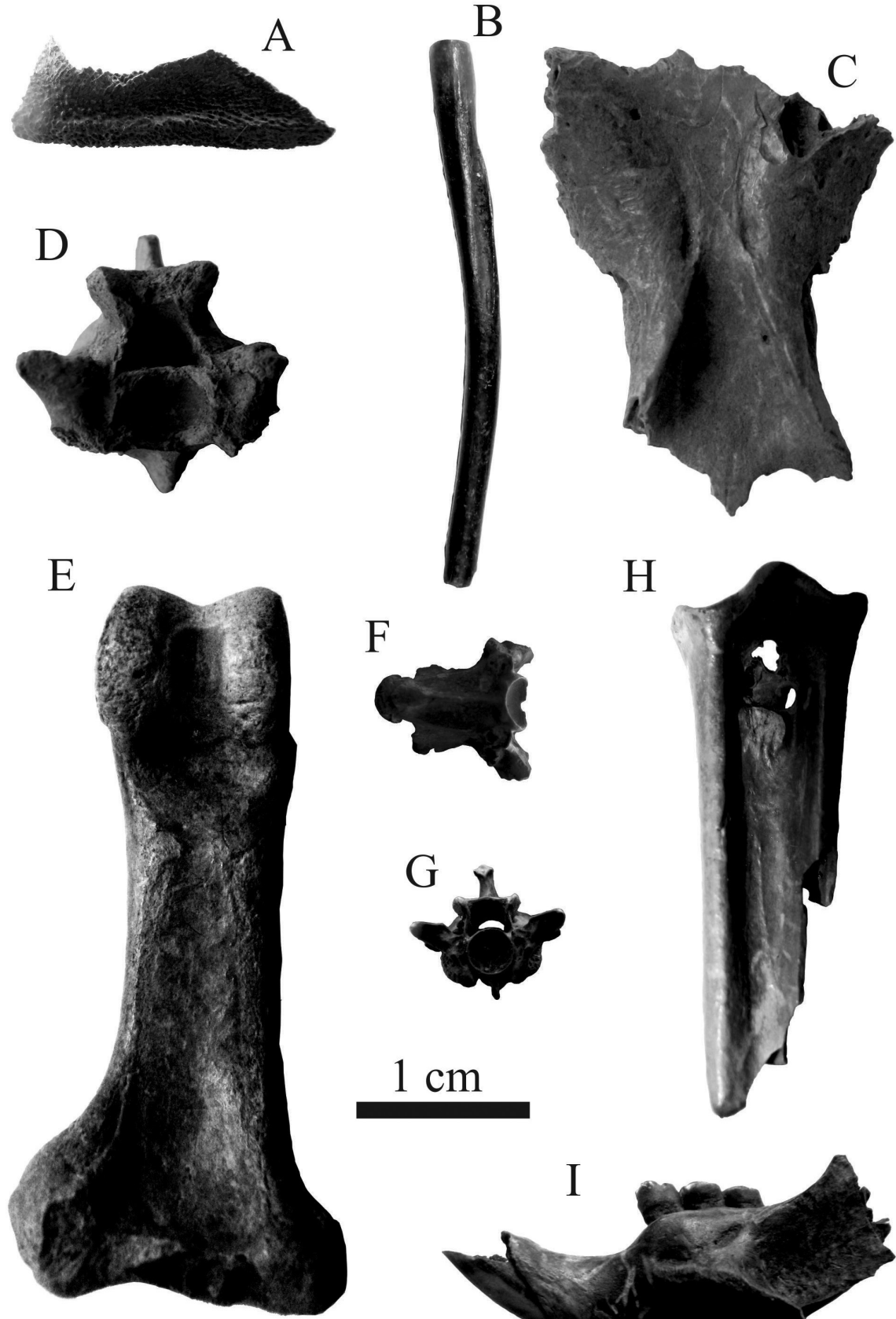

H
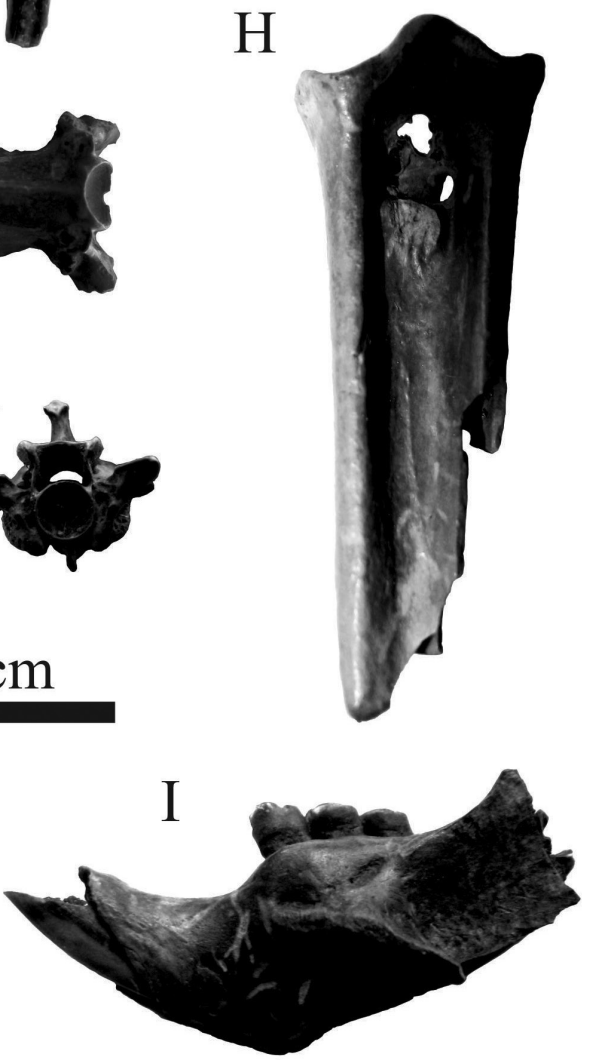

Figura 1. Nuevos registros fósiles de vertebrados terrestres para Las Llanadas, Yaguajay, Sancti Spíritus, Cuba. A: Peltophryne cf. empusus, maxila izquierda (vista labial); B: Osteopilus septentrionalis, fémur (vista lateral); C: Cyclura sp., frontal (vista ventral); D: Chilabothrus sp., vértebra (vista proximal); E: Buteogallus sp., falange (vista ventral); F: Cubophis sp., vértebra (vista ventral); G: Tropidophis sp., vértebra (vista proximal); H: Tyto alba, tarsometatarso (vista frontal); I: Boromys ofella, hemimandíbula izquierda (vista lateral). 
TABLA I.

Cantidad de restos identificados en el depósito Las Llanadas, Yaguajay, Sancti Spíritus, Cuba, organizados hasta la categoría de familia.

\begin{tabular}{|c|c|c|c|c|}
\hline Clase & Orden & Familia & No. restos & Porcentaje \\
\hline \multirow{3}{*}{ Amphibia } & \multirow{3}{*}{ Anura } & Bufonidae & 1 & 0.1 \\
\hline & & Hylidae & 2 & 0.2 \\
\hline & & Ranidae & 1 & 0.1 \\
\hline \multirow[t]{6}{*}{ Reptilia } & Testudines & Testudinidae & 84 & 9.2 \\
\hline & Crocodylia & Crocodylidae & 39 & 4.5 \\
\hline & \multirow{4}{*}{ Squamata } & Iguanidae & 2 & 0.2 \\
\hline & & Dipsadidae & 1 & 0.1 \\
\hline & & Boidae & 8 & 0.9 \\
\hline & & Tropidophiidae & 2 & 0.2 \\
\hline \multirow[t]{2}{*}{ Aves } & Strigiformes & Tytonidae & 1 & 0.1 \\
\hline & Falconiformes & $\begin{array}{l}\text { Accipitridae } \\
\text { Indet. }\end{array}$ & $\begin{array}{l}2 \\
1\end{array}$ & $\begin{array}{l}0.2 \\
0.1\end{array}$ \\
\hline \multirow[t]{5}{*}{ Mammalia } & Chiroptera & Indet. & 1 & 0.1 \\
\hline & \multirow{2}{*}{ Rodentia } & Echimyidae & 1 & 0.1 \\
\hline & & Capromyidae & 28 & 3.2 \\
\hline & Pilosa & Megalonychidae & 698 & 80 \\
\hline & & Total & 872 & \\
\hline
\end{tabular}

Indet: indeterminado.

\section{CONCLUSIONES}

Además de los huesos grandes extraídos por Carlos de la Torre y Barnum Brown, en el depósito existen otros huesos pequeños también con mucha importancia para la reconstrucción paleoecológica. Con la identificación de anuros, reptiles, aves y otro mamífero, queda más completa la lista de la fauna cuaternaria regional.

\section{AGRADECIMIENTOS}

Agradecemos a Luis M. Díaz (MNHNC) por su ayuda con la identificación, y por préstamos de material comparativo. A Adonis González y Lisbet Barban por permitirnos el acceso a la colección del IES. Agradecemos a los revisores del manuscrito por sus valiosas contribuciones. 


\section{LITERATURA CITADA}

Brown, B. 1913. Some Cuban fossils: a hot spring yields up the bones animals that lived before the Advent of Man. The Journal of the American Museum, 13: 221-228.

Fernández-López, S. R. 2000. Temas de Tafonomía, Madrid, Departamento de Paleontología, Facultad de Ciencias Geológicas, Universidad Complutense de Madrid, 167p.

Holz, M. y Barberena, M. C. 1994. Taphonomy of the south Brazilian Triassic paleoherpetofauna: pattern of death, transport and burial Palaeogeography, Palaeoclimatology, Palaeoecology, 107: 179-197.

Martínez-López, J. G. 2013. Caracterización geográfica del área paleontológica "Las Llanadas" (Sancti Spíritus, Cuba), mediante el uso de las tecnologías de la Geomática. Tesina, Instituto de Geografía Tropical, La Habana, Cuba. 57p.

Matthew, W. D. 1931. Genera and new species of ground sloth from the Pleistocene of Cuba. American Museum Novitates, 511: 1-5.

Torre, C. H. 1910. Excursión a la Sierra de Jatibonico: osamentas fósiles de Megalocnus rodens o Myomorphus cubensis: comprobación de la naturaleza continental de Cuba a principios de la época Cuaternaria. Anales de la Academia de Ciencias Médicas, Físicas y Naturales de la Habana, 47: 204-217. 


\section{Novitates Caribaea}

\section{Instrucciones a los autores}

Novitates Caribaea es una de las revistas científicas del Museo Nacional de Historia Natural "Prof. Eugenio de Jesús Marcano", destinada a publicar artículos originales en zoología, paleobiología y geología, pudiendo cubrir áreas como: sistemática, taxonomía, biogeografía, evolución, genética, biología molecular, embriología, comportamiento y ecología. El Comité Editorial, en la selección de los trabajos sometidos, dará prioridad a los que traten sobre la biodiversidad y la historia natural de La Hispaniola y el Caribe. Su salida será anual. Se aceptarán trabajos en Español o en Inglés (si esta es la primera lengua del autor), debiendo incluir Resumen en ambos idiomas. Los mismos serán enviados en versión electrónica (Microsoft Word) a las direcciones especificadas al final de la página.

Los dibujos deberán estar hechos en papel blanco y con tinta negra, bien definidos y ensamblados en láminas en caso de ser varios y así requerirlo el trabajo. Todas las figuras se enviarán también por correo electrónico, en extensión o formato BMP o JPG y con resolución de 270-300 DPI. La indicación de escala deberá incluirse tanto en los dibujos como en las fotos.

Formato requerido. Los artículos científicos sometidos se ajustarán a la siguiente conformación:

1) Título del trabajo (Español e Inglés) y nombre del autor o los autores con su dirección de correo electrónico, así como el nombre y la dirección de la institución para la que laboran o a la que están asociados (si es el caso)

2) Resumen y Palabras Clave (en Español e Inglés)

3) Introducción

4) Objetivos

5) Materiales y Métodos

6) Resultados. Bajo este epígrafe los autores podrán incluir otros subtítulos de acuerdo a las características del trabajo sometido, dándole a estos el ordenamiento que entiendan pertinente

a) En trabajos de descripción de táxones nuevos para la ciencia, los autores deberán incluir en sus Resultados los siguientes acápites: Diagnosis (en ambos idiomas), Descripción, Tipos (indicando localidades, colectores, fechas de colecta y colecciones o instituciones de destino) y Etimología (dando cuenta de los nombres nuevos). La inclusión en Resultados de otros bloques de contenido, tales como Historia Natural y Comentario, es opcional. El nombre del taxon nuevo deberá señalarse con las inscripciones sp. nov. o gen. nov., según el caso, cada vez que aparezca en el texto. Todos los nombres genéricos y específicos deberán aparecer en itálicas, pudiendo abreviarse a partir de su primera referencia en el texto mediante la letra inicial del género seguida de un punto y el adjetivo específico (ejemplo: Achromoporus heteromus...A. heteromus). En sentido general, para los nombres y todos los actos nomenclaturales, los autores y los editores se regirán por las normas establecidas en la última edición que esté vigente del Código Internacional de Nomenclatura Zoológica elaborado por la Comisión Internacional de Nomenclatura Zoológica.

b) Las citas en el texto se harán de la siguiente manera: Gutiérrez (2013) o (Gutiérrez, 2013); Bonato et al. (2011) o (Bonato et al., 2011), estos últimos dos ejemplos en caso de ser más de dos autores.

7) Discusión (si aplica en el caso de descripción de nuevas especies). Este acápite podría fusionarse con el de Resultados presentándolo como Resultados y Discusión

8) Conclusiones (si aplica en el caso de descripción de nuevas especies)

9) Agradecimientos (opcional)

10) Literatura Citada. Este acápite se escribirá de acuerdo a los números anteriores de esta revista y de Hispaniolana. Ejemplos:

Nelson, G. 1989. Cladistics and evolutionary models. Cladistics, 5: 275-289.

Si son dos o más autores de una publicación escrita en Español se usará la conjunción “y” en la Literatura Citada, aunque la publicación referida esté originalmente en Inglés; si la publicación nos la someten en Inglés, en la Literatura Citada correspondiente se usará "and" en lugar de "y".

Mauries, J. P. y R. L. Hoffman. 1998. On the identity of two enigmatic Hispaniolan millipeds (Spirobolida: Rhinocricidae). Myriapodologica, 5 (9): 95-102.

Perez-Gelabert, D. E. and G. D. Edgecombe. 2013. Scutigeromorph centipedes (Chilopoda: Scutigeromorpha) of the Dominican Republic, Hispaniola. Novitates Caribaea, 6: 36-44.

Aceptaremos otros tipos de colaboraciones como Notas Científicas y Revisión de Libros, las cuales no se ajustarán a todas las normas establecidas arriba, quedando su conformación, en cada caso, sometida a la deliberación entre los autores y los editores. El Comité Editorial de Novitates Caribaea revisará los trabajos sometidos y los enviará a los correspondientes revisores según el tema. Los resultados de la revisión se darán a conocer a los autores previamente a la aceptación definitiva del trabajo. La publicación se hará sin costo, recibiendo los autores un ejemplar del número de la revista y una versión electrónica en formato PDF de su artículo. Las direcciones de correo electrónico a las que se enviarán los trabajos son: "Celeste Mir" c.mir@mnhn.gov.do y "Carlos Suriel” c.suriel@mnhn.gov.do. Para otros tipos de comunicación: Museo Nacional de Historia Natural "Prof. Eugenio de Jesús Marcano". Calle César Nicolás Penson, Plaza de la Cultura Juan Pablo Duarte, Santo Domingo (10204), República Dominicana. Teléfono: (809) 689 0106. Fax: (809) 6890100. 